\title{
Hábitos de Leitura de Estudantes do Curso de Fonoaudiologia
}

\author{
Ana Paula Seixas Gomes ${ }^{1}$, Patrícia Pupin Mandrá* \\ ${ }^{1}$ Bolsista do Programa Ensinar com Pesquisa da Pró-Reitoria de Graduação da Universidade de São Paulo
}

* Autora para correspondência: ppmandra@fmrp.usp.br

\section{RESUMO}

O presente artigo tem como objetivo verificar o hábito de leitura de estudantes da graduação em Fonoaudiologia da FMRP-USP. Para tanto, realizou-se um estudo do qual participaram voluntariamente 114 alunos matriculados no referido curso a partir do segundo semestre do ano de 2014. Os participantes responderam a um questionário escalar elaborado para esta pesquisa. Os resultados foram organizados em tabelas do Excel for Word ${ }^{\circledR}$ para o cálculo da frequência absoluta e relativa de acordo com o período de graduação $\left(2^{\circ}, 4^{\circ}, 6^{\circ}\right.$ e $8^{\circ}$ semestres). Observou-se que dos 114 participantes que constituíram a amostra, 56,1\% cursaram o ensino fundamental (de $1^{\mathrm{a}}$ a $4^{\mathrm{a}}$ série) em escolas públicas e as séries seguintes (da $5^{\mathrm{a}}$ série ao colegial) em escolas particulares $(57,9 \%$ da $5^{\mathrm{a}}$ à $8^{\mathrm{a}}$, e $63,16 \%$ no ensino médio). O principal meio de atualização citado foi a internet $(65,6 \%)$. Uma ou duas horas foi o tempo dedicado à leitura para 37,7\% dos participantes. $\mathrm{O}$ acesso foi considerado raro para e-books $(42,9 \%)$, dissertações/teses na base da biblioteca virtual da USP $(52,6 \%)$ e revistas eletrônicas $(48,85 \%)$. O hábito de leitura foi avaliado como crucial para: formação pessoal, desempenho acadêmico, elaboração da escrita e desenvolvimento do raciocínio. Manter o hábito de leitura cursando a graduação em tempo integral raramente é possível para 51,7\% dos entrevistados, o que se confirmou quando $47,4 \%$ destacaram a disponibilidade de tempo como uma das estratégias para melhorar o hábito de leitura. Por fim, concluiu-se que o hábito de leitura foi considerado crucial pelos estudantes, no entanto, manter esse hábito durante a graduação é o desafio devido à dificuldade, relatada pelos estudantes, em organizar o tempo disponível.

Palavras-Chave: Leitura; Hábitos; Formação Pessoal.

\begin{abstract}
The aim of this article is to check the reading habits of students of Speech Therapy at FMRP-USP. Therefore, a study was carried out in which 114 students enrolled in this course in the 2nd semester of 2014. Participants answered a questionnaire scale developed for this study. The results were organized in tables Excel for Word $\mathbb{R}$ to calculate the absolute and relative frequency according to the graduation period (2, 4, 6 and 8 semesters). It was observed that of the 114 participants who constituted the sample, $56,1 \%$ have finished elementary school (1st to 4 th grade) in public education and the following series (5th grade to high school) in private schools (57,9\% of 5th to 8 th and $63,16 \%$ in high school). The primary means of cited update was internet $(65,6 \%)$. One or two hours was the time dedicated reading to $37,7 \%$ of the participants. Access was considered rare for e-books $(42,9 \%)$, dissertations / theses on the basis of the virtual library of USP $(52,6 \%)$ and electronic journals $(48,85 \%)$. The reading habit was assessed as crucial for: personal formation, academic performance, writing elaboration and development of reasoning. Keep reading habit attending full-time undergraduate is rarely possible for 51,7\% of respondents, which was confirmed when $47,4 \%$ highlighted the availability of time as one of the strategies to improve the reading habit. Finally, it was concluded that the reading habit was considered crucial by the students, however, maintain this habit during graduation is the challenge because of the difficulty, reported by students to organize the available time.
\end{abstract}

Keywords: Reading; Habits; Personal Formation.

\section{Introdução}

A leitura é vista como uma competência básica que todos deveriam adquirir para poderem aprender, trabalhar e realizar-se no mundo contemporâneo (SILVEIRA, 2010). Quanto maiores a proficiência e a prática do ato da leitura, melhor o desempenho em vários aspectos da vida acadêmica e social (FRANCO \& MOLINARI, 
2013). Grande parte dos conhecimentos é obtida através dela, o que possibilita ampliar e aprofundar o conhecimento em determinada área (LAKATOS \& MARCONI, 2007). Depois da leitura somos capazes de saber algo que não sabíamos, ter algo que não tínhamos e ser algo que não éramos (BERTICELLI \& SCHIAVINI, 2013).

Para o ingresso no mercado de trabalho e para o exercício da cidadania, a leitura é considerada como imprescindível, porém pesquisas e avaliações educacionais nacionais apontam para a precária formação de um público leitor no Brasil (SILVEIRA, 2010). Um bom nível de compreensão leitora dependerá de variáveis como as condições do texto e do leitor no momento da leitura (OLIVEIRA \& SANTOS, 2003), as condições de leitura, dos momentos e o tempo que lhe são concedidos, e do papel simbólico que lhe é atribuído. $\mathrm{O}$ amor pela leitura não é um dom inato, mas um exercício que se transforma em necessidade na medida em que é incorporado como hábito (MAZZA, 2013).

O leitor contemporâneo pode ter acesso a fatos em tempo simultâneo com seu acontecer (BERTICELLI \& SCHIAVINI, 2013), pois estão disponíveis inúmeros meios para armazenar e acessar aquilo que antes somente o livro impresso disponibilizava (OSWALD \& ROCHA, 2013). O texto ganhou velocidade, com a informação correndo em tempo real pela internet (BERTICELLI \& SCHIAVINI, 2013). O acesso em casa, e/ou via dispositivos móveis (telefones celulares, redes $3 \mathrm{G}$, wi-fi), estimula as pessoas não só a lerem e a escreverem num ritmo intenso e acelerado, como também incentiva a circulação de leituras e escritas por intermédio da conexão em redes abertas, como ocorre nas redes sociais: Facebook, MySpace, Ning, Twitter (OSWALD \& ROCHA, 2013).

A leitura está em todos os lugares, todos os contextos, todos os cursos e em todas as disciplinas (BRIDON \& NEITZEL, 2014). Assim, deveria ser a preocupação mais importante na Universidade (HUSSEIN, 2008), pois é fundamental para a formação social e cognitiva dos estudantes, qualificando-os para a inserção no mercado de trabalho. É importante saber ler criticamente, não somente para recuperar a informação acumulada historicamente, mas também para utilizá-la visando a uma prática profissional eficiente (CARAVANTES, 2006). O hábito de leitura no dia a dia do graduando fomenta o conhecimento e promove a construção de uma sociedade com indivíduos mais bem preparados no que diz respeito a praticarem os seus direitos como cidadãos de maneira ativa (PIRES, 2012).

A busca pelo conhecimento deve estar presente cotidianamente na vida dos estudantes, para que sua formação acadêmica seja plena. Todos, em maior ou menor grau, têm necessidade de informação para continuarem o processo de sua formação educacional (CARDOSO, FRANÇA \& MARIANI, 2014).

A internet abriu a possibilidade de um acesso massivo de baixo custo ao conteúdo dos periódicos por meio digital (ORTELLADO, 2008). Bancos e bases de dados digitais são ferramentas importantíssimas para os usuários de conhecimento científico. Armazenados em ambientes virtuais, especialmente na Web, eles agrupam rica fonte de informação, assumindo diferenciadas tipologias, nos níveis nacional e internacional, disponibilizando textos completos. Os sítios oferecem ao usuário uma série de recursos que o auxiliam a realizar suas pesquisas, a utilizar gerenciadores de referência, a promover a disseminação seletiva da informação, a obter informação sobre índice de citação de artigos e fatores de impacto em publicações científicas (CARDOSO, FRANÇA \& MARIANI, 2014). As revistas eletrônicas estão disponíveis na $W e b$ em diferentes fontes de informações, como: portais, índices, bases de dados, repositórios e diretórios (FERREIRA \& CAREGNATO, 2014).

O Portal de Periódicos da Capes, fundado em 2000, é uma iniciativa pública que subsidia o acesso à produção científica para pesquisadores nas universidades brasileiras. Ele possui um dos maiores acervos mundiais nesse setor (ROHENKOHL, 2014) e permite acesso rápido a conteúdos científicos internacionais de qualidade, proporcionando maior igualdade de oportunidades para os pesquisadores de todas as regiões do Brasil, sendo 
parte fundamental e indispensável no avanço da pesquisa acadêmica brasileira (GONZÁLEZ, GOMES \& ANDRADE, 2014; ROHENKOHL, 2014). O governo federal do Brasil foi pioneiro ao implantar um portal de comunicação científica de acesso livre, o Scielo, criado em 1996. Nele se fazem presentes arquivos digitais de vários países, incluindo periódicos e livros (GONZÁLEZ, GOMES \& ANDRADE, 2014).

Para despertar o gosto pela leitura e estimular a autonomia, é necessário ter em mente a diversidade humana, considerar as idades, os estágios do desenvolvimento, as características próprias de cada grupo, o gosto e o ritmo próprios de cada pessoa. Os projetos de leitura devem rejeitar tentações de modelo único. Exigem uma atitude aberta, flexível onde caibam múltiplos percursos, os percursos que a diversidade humana aconselha a respeitar (SILVEIRA, 2010).

A motivação do aluno em relação ao seu aprendizado é muito importante no momento do estudo (WATKINS \& COFFEY, 2004). Pesquisa realizada em 1996 já demonstrava que os universitários não tinham tempo para se dedicarem ao estudo (LARA CAMPOS, SILVA FILHO, GAMPOS \& ROCHA, 1996). Já em 2007 o resultado de outra pesquisa indicou que a leitura de livros não obrigatórios à aprendizagem acadêmica não era feita por boa parte dos participantes e que raramente liam jornais (OLIVEIRA \& OLIVEIRA, 2007).

$\mathrm{Na}$ atualidade, ser leitor exige do aluno motivação, vontade, esforço, pois muitas são as funções desempenhadas pela leitura na escola e na vida. Lêse para ampliar os limites do conhecimento, para obter informação, para descontrair, para refletir. A aprendizagem da leitura é para a vida inteira. Uma vez que a sociedade confere muita importância à leitura, a integração do indivíduo exige a aquisição e o desenvolvimento de competências relacionadas com a compreensão da informação escrita. Fica assim reforçada a importância da leitura como ferramenta de aprendizagem fundamental para todos, em todas as idades (MARTINS \& SÁ, 2008). Deve haver escolas que saibam formar leitores, valendose de mediadores bem formados e de múltiplas estratégias/recursos para alcançar essa finalidade (SILVEIRA, 2010).

A proposta desta pesquisa foi discutir os hábitos de leitura de estudantes de Fonoaudiologia, uma vez que os conteúdos programáticos das disciplinas do curso trazem bibliografias obrigatórias, ou seja, referências de livros e artigos, entre outros textos que o aluno deverá acessar para construir seu conhecimento sobre a temática da disciplina. Conhecer os hábitos de leitura dos estudantes na era digital é fundamental para que os docentes possam buscar novas estratégias de incentivo à leitura acadêmica e geral, uma vez que se pretende formar um fonoaudiólogo com visão crítica e reflexiva, preparado para compreender sua atuação profissional no contexto social, político e de saúde nacional.

Portanto, pretendeu-se verificar o hábito de leitura de estudantes do curso de Fonoaudiologia e discutir estratégias para incentivar esse hábito durante a formação acadêmica.

\section{Método}

O estudo foi aprovado pelo Comitê de Ética em Pesquisa do Hospital das Clínicas da Faculdade de Medicina de Ribeirão Preto, da Universidade de São Paulo (HCFMRP-USP), parecer n. 2685/2014, após autorização da Comissão de Graduação da FMRP-USP, ofício n. 061 - SVGRDA/ FMRP/20.03.2014, em acordo com a RESOLUÇÃO n. 466, de 12 de dezembro de 2012.

Participaram voluntariamente do estudo 114 alunos matriculados no curso de Fonoaudiologia, sendo 28 mulheres do segundo semestre com média de idade de 19,2 anos, 30 mulheres e um homem do quarto semestre cuja média era de 19,7 anos, 32 mulheres e um homem do sexto semestre com média de 20,6 anos e 22 mulheres do oitavo semestre com média de 21 anos. Todos os participantes tinham entre dezessete e 31 anos de idade.

Após a autorização do presidente da Comissão de Graduação da FMRP-USP e do Comitê de Ética em Pesquisa do Hospital das Clínicas da Faculdade de Medicina de Ribeirão Preto, da Universidade de São Paulo (HCFMRP-USP), o orientador/docente obteve, através do sistema 


\begin{tabular}{|c|c|c|c|c|c|c|c|c|c|c|}
\hline \multirow{2}{*}{$\begin{array}{l}\text { Participantes } \\
\text { Sexo }\end{array}$} & \multicolumn{2}{|c|}{$2^{a}$ etapa } & \multicolumn{2}{|c|}{$4^{a}$ etapa } & \multicolumn{2}{|c|}{$6^{a}$ etapa } & \multicolumn{2}{|c|}{$8^{a}$ etapa } & \multicolumn{2}{|c|}{ Total } \\
\hline & $\mathrm{n}$ & $\%$ & $\mathrm{n}$ & $\%$ & $\mathrm{n}$ & $\%$ & $\mathrm{n}$ & $\%$ & $\mathrm{n}$ & $\%$ \\
\hline Feminino & 28 & 100 & 30 & 96,8 & 32 & 97 & 22 & 100 & 112 & 98,25 \\
\hline Masculino & 0 & 0 & 1 & 3,2 & 1 & 3 & 0 & 0 & 2 & 1,75 \\
\hline Trabalham & 1 & 3,6 & 1 & 3,2 & 2 & 6 & 1 & 4,5 & 5 & 4,4 \\
\hline Escolaridade & $\mathrm{n}$ & $\%$ & $\mathrm{n}$ & $\%$ & $\mathrm{n}$ & $\%$ & $\mathrm{n}$ & $\%$ & $\mathrm{n}$ & $\%$ \\
\hline $\begin{array}{l}\text { Ensino Médio } \\
\text { Público }\end{array}$ & $9^{* *}$ & 32,2 & 6 & 19,35 & $7^{*}$ & 21,2 & 8 & 36,4 & 30 & 26,3 \\
\hline $\begin{array}{l}\text { Ensino Médido } \\
\text { Privado }\end{array}$ & 14 & 50 & 21 & 67,7 & 23 & 69 & 14 & 63,6 & 72 & 63,1 \\
\hline $\begin{array}{l}\text { Ensino Médio } \\
\text { (Técnico) } \\
\text { Público }\end{array}$ & 1 & 3,6 & 3 & 9,7 & 1 & 3 & 0 & 0 & 5 & 4,4 \\
\hline $\begin{array}{l}\text { Ensino Médio } \\
\text { (Técnico) } \\
\text { Privado }\end{array}$ & 0 & 0 & 1 & 3,2 & 1 & 3 & 0 & 0 & 2 & 1,75 \\
\hline
\end{tabular}

Tabela 1 - Caracterização dos participantes. *Um participante não respondeu a questão. ** Dois participantes não responderam.

Fúpiter WEB-Graduação USP, a lista de todos os alunos matriculados no Curso. Os alunos foram convidados, pelo aluno/pesquisador, a participar e, após a assinatura do termo de consentimento livre esclarecido (TCLE), receberam o questionário composto por 25 questões de múltipla escolha e duas discursivas, relacionadas ao hábito de leitura e ao acesso a material de leitura. Procedeu-se à coleta nos momentos de intervalo entre as atividades acadêmicas, nas dependências do bloco didático da instituição, locais onde os alunos realizavam suas atividades, onde estava disponível uma sala com cadeiras.

Os questionários respondidos foram lidos e separados por período de graduação $\left(2^{\circ}, 4^{\circ}, 6^{\circ}\right.$ e $8^{\circ}$ semestres). Os resultados foram organizados em tabelas do Excel ${ }^{\circledR}$ para o cálculo da frequência absoluta e relativa. Posteriormente, reagrupou-se em tabelas o conjunto de dados para a apresentação dos resultados finais.

\section{Resultados}

A maioria dos participantes eram do sexo feminino $(\mathrm{n}=112), 63,1 \%$ cursaram o ensino médio em escola privada. A escolaridade paterna era, predominantemente, ensino médio e superior completo, $35,97 \%$ e $35,0 \%$, respectivamente. E materna, $30,7 \%$ com ensino médio completo e $42,1 \%$ com superior completo.

A tabela 2 mostra que o percentual de participantes que tinham lido no máximo dois livros era de 41,2\%, sendo 54,4\% de obras literárias de ficção. O principal meio de atualização citado foi a internet, com o acesso a sites de agências de notícias $(65,6 \%)$. A leitura não era realizada com frequência: 34,2\% liam algumas vezes por semana, e 32,4\% raramente o faziam. $\mathrm{O}$ mesmo ocorreu com a frequência de manipulação de livros (47,4\% manipulam livros algumas vezes por semana). As visitas à biblioteca do campus e a livrarias foram raras, com respectivamente 50,9\% e 58,8\%. Quanto ao tempo 


\begin{tabular}{|c|c|c|c|c|c|c|c|c|c|c|}
\hline Etapa & \multicolumn{2}{|c|}{$2^{a}$} & \multicolumn{2}{|c|}{$4^{a}$} & \multicolumn{2}{|c|}{$6^{a}$} & \multicolumn{2}{|c|}{$8^{a}$} & \multicolumn{2}{|c|}{ Total } \\
\hline Total & \multicolumn{2}{|c|}{28} & \multicolumn{2}{|c|}{31} & \multicolumn{2}{|c|}{33} & \multicolumn{2}{|c|}{22} & \multicolumn{2}{|c|}{114} \\
\hline $\begin{array}{l}\text { Quantidade de livros } \\
\text { lidos no ano* }\end{array}$ & $\mathrm{n}$ & $\%$ & $\mathrm{n}$ & $\%$ & $\mathrm{n}$ & $\%$ & $\mathrm{n}$ & $\%$ & $\mathrm{n}$ & $\%$ \\
\hline Nenhum & 5 & 17,85 & 5 & 16,1 & 7 & 21,2 & 6 & 27,3 & 23 & 20,17 \\
\hline No máximo dois & 9 & 32,1 & 17 & 54,8 & 11 & 33,3 & 10 & 45,45 & 47 & 41,2 \\
\hline Entre três e cinco & 12 & 42,85 & 8 & 25,8 & 12 & 36,36 & 4 & 18,2 & 36 & 31,6 \\
\hline Entre seis e oito & 1 & 3,6 & 1 & 3,2 & 2 & 6 & 1 & 4,6 & 5 & 4,4 \\
\hline Mais de oito & 0 & 0 & 0 & 0 & 1 & 3 & 1 & 4,6 & 2 & 1,75 \\
\hline Tipos de livros que mais lê & $\mathrm{n}$ & $\%$ & $\mathrm{n}$ & $\%$ & $\mathrm{n}$ & $\%$ & $\mathrm{n}$ & $\%$ & $\mathrm{n}$ & $\%$ \\
\hline Obras literárias de ficção & 18 & 64,3 & 21 & 67,7 & 12 & 36,36 & 11 & 50 & 62 & 54,4 \\
\hline Obras literárias de não ficção & 5 & 17,85 & 9 & 29 & 7 & 21,2 & 6 & 27,3 & 27 & 23,7 \\
\hline Livros técnicos & 1 & 3,6 & 2 & 6,45 & 8 & 24,3 & 5 & 22,8 & 16 & 14 \\
\hline Livros de autoajuda & 1 & 3,6 & 1 & 3,2 & 2 & 6 & 3 & 13,6 & 7 & 6,1 \\
\hline Outros & $4^{* *}$ & 14,3 & 1 & 3,2 & 6 & 18,2 & 2 & 9 & 13 & 11,4 \\
\hline $\begin{array}{l}\text { Meios utilizados para se } \\
\text { manter atualizado }\end{array}$ & $\mathrm{n}$ & $\%$ & $\mathrm{n}$ & $\%$ & $\mathrm{n}$ & $\%$ & $\mathrm{n}$ & $\%$ & $\mathrm{n}$ & $\%$ \\
\hline Jornais impressos & 2 & 7,1 & 1 & 3,2 & 2 & 6 & 1 & 4,6 & 6 & 57,9 \\
\hline Revistas impressas & 1 & 3,6 & 0 & 0 & 2 & 6 & 1 & 4,6 & 4 & 3,5 \\
\hline TV & 10 & 35,7 & 5 & 16,1 & $8^{* * *}$ & 24,3 & 10 & 45,45 & 33 & 28,9 \\
\hline Rádio & 0 & 0 & 1 & 3,2 & 0 & 0 & 2 & 9 & 3 & 2,6 \\
\hline $\begin{array}{l}\text { Internet (Site de } \\
\text { relacionamento) }\end{array}$ & 18 & 64,3 & 13 & 49 & 15 & 45,45 & 6 & 27,3 & 52 & 45,6 \\
\hline $\begin{array}{l}\text { Internet (Site de agência de } \\
\text { notícias) }\end{array}$ & 17 & 60,7 & 23 & 74,1 & 21 & 63,6 & 13 & 59 & 74 & 65,6 \\
\hline Frequência de Leitura & $\mathrm{n}$ & $\%$ & $\mathrm{n}$ & $\%$ & $\mathrm{n}$ & $\%$ & $\mathrm{n}$ & $\%$ & $\mathrm{n}$ & $\%$ \\
\hline Diariamente & 6 & 21,4 & 5 & 16,1 & $8^{* * *}$ & 24,3 & 2 & 9 & 21 & 18,4 \\
\hline Algumas vezes por semana & 5 & 17,85 & 11 & 35,5 & 14 & 42,42 & 9 & 40,9 & 39 & 34,2 \\
\hline Somente aos fins de semana & 5 & 17,85 & 6 & 19,35 & 2 & 6 & 2 & 9 & 15 & 13,16 \\
\hline Raramente & 11 & 39,3 & 9 & 29 & 8 & 24,3 & 9 & 40,9 & 37 & 32,4 \\
\hline Nunca & 0 & 0 & 0 & 0 & 0 & 0 & 0 & 0 & 0 & 0 \\
\hline
\end{tabular}




\begin{tabular}{|c|c|c|c|c|c|c|c|c|c|c|}
\hline $\begin{array}{l}\text { Frequência com que } \\
\text { manipula livros }\end{array}$ & $\mathrm{n}$ & $\%$ & $\mathrm{n}$ & $\%$ & $\mathrm{n}$ & $\%$ & $\mathrm{n}$ & $\%$ & $\mathrm{n}$ & $\%$ \\
\hline Diariamente & 6 & 21,4 & 2 & 6,45 & 6 & 18,2 & 4 & 18,2 & 18 & 15,8 \\
\hline Algumas vezes por semana & 10 & 35,7 & 13 & 49,9 & 19 & 57,6 & 12 & 54,6 & 54 & 47,4 \\
\hline Somente aos fins de semana & 5 & 17,85 & 6 & 19,35 & 2 & 6 & 1 & 4,6 & 14 & 12,3 \\
\hline Raramente & 6 & 21,4 & 9 & 29 & 6 & 18,2 & 5 & 22,7 & 18 & 15,8 \\
\hline Nunca & 13,6 & 13,6 & 0 & 0 & 0 & 0 & 0 & 0 & 1 & 0,9 \\
\hline $\begin{array}{l}\text { Frequência com que vai } \\
\text { à biblioteca central do } \\
\text { campus para consultar } \\
\text { material impresso }\end{array}$ & $\mathrm{n}$ & $\%$ & $\mathrm{n}$ & $\%$ & $\mathrm{n}$ & $\%$ & $\mathrm{n}$ & $\%$ & $\mathrm{n}$ & $\%$ \\
\hline Diariamente & 0 & 0 & 0 & 0 & 0 & 0 & 0 & 0 & 0 & 0 \\
\hline Algumas vezes por semana & 17 & 60,7 & 11 & 35,5 & 12 & 36,7 & 7 & 21,2 & 47 & 41,2 \\
\hline Somente aos fins de semana & 0 & 0 & 1 & 3,2 & 0 & 0 & 2 & 9 & 3 & 2,6 \\
\hline Raramente & 9 & 32,1 & 17 & 54,8 & 19 & 57,6 & 13 & 59 & 58 & 50,9 \\
\hline Nunca & 2 & 7,1 & 2 & 6,45 & 2 & 6 & 0 & 0 & 6 & 5,3 \\
\hline $\begin{array}{l}\text { Frequência com que vai } \\
\text { a uma livraria }\end{array}$ & $\mathrm{n}$ & $\%$ & $\mathrm{n}$ & $\%$ & $\mathrm{n}$ & $\%$ & $\mathrm{n}$ & $\%$ & $\mathrm{n}$ & $\%$ \\
\hline Diariamente & 0 & 0 & 0 & 0 & 0 & 0 & 0 & 0 & 0 & 0 \\
\hline Algumas vezes por semana & 0 & 0 & 1 & 3,2 & 5 & 15,1 & 3 & 13,6 & 9 & 7,9 \\
\hline Somente aos fins de semana & 13 & 46,4 & 7 & 22,6 & 10 & 30,3 & 6 & 27,3 & 36 & 31,6 \\
\hline Raramente & 15 & 53,6 & 22 & 70,9 & 17 & 51,5 & 13 & 59 & 67 & 58,8 \\
\hline Nunca & 0 & 0 & 1 & 3,2 & 0 & 0 & 0 & 0 & 1 & 0,9 \\
\hline $\begin{array}{l}\text { Horas dedicadas à leitura } \\
\text { na última semana }\end{array}$ & $\mathrm{n}$ & $\%$ & $\mathrm{n}$ & $\%$ & $\mathrm{n}$ & $\%$ & $\mathrm{n}$ & $\%$ & $\mathrm{n}$ & $\%$ \\
\hline Nenhuma & 4 & 14,3 & 5 & 16,1 & 1 & 3 & 1 & 4,6 & 11 & 9,65 \\
\hline Uma a duas & 14 & 50 & 15 & 48,4 & 10 & 30,3 & 4 & 12,1 & 43 & 37,7 \\
\hline Três a cinco & 3 & 10,7 & 10 & 32,25 & 13 & 39,4 & 8 & 36,4 & 34 & 29,8 \\
\hline Seis a oito & 6 & 21,4 & 0 & 0 & 4 & 12,1 & 3 & 13,6 & 13 & 11,4 \\
\hline Mais de oito & 1 & 3,6 & 1 & 3,2 & 5 & 15 & 6 & 27,3 & 13 & 11,4 \\
\hline
\end{tabular}

Tabela 2 - Hábitos de leitura. Legenda: *Exceto os da graduação **um participante respondeu que não lê ***um participante não respondeu. 
dedicado à leitura, 37,7\% dos participantes havia lido durante uma ou duas horas na última semana.

$\mathrm{O}$ acesso a $e$-book foi classificado como raro $(42,9 \%)$ ou nunca $(31,6 \%)$. Artigos científicos indexados pelo Scielo tinham sido acessados algumas vezes por semana por 49,1\%, sendo que os participantes da $8^{\mathrm{a}}$ etapa realizaram o maior número de acessos (81,8\%). O acesso a dissertações/teses na base da biblioteca virtual da USP foi raro $(52,6 \%)$. O mesmo ocorre com as revistas eletrônicas: 48,85\% raramente as acessavam, e $38,6 \%$ nunca o faziam. Os acessos ao portal de periódicos da Capes foram classificados como raros $(45,6 \%)$ ou nunca aconteciam $(45,6 \%)$. Em relação à bibliografia obrigatória das disciplinas do curso, 30,7\% dos participantes referiram tê-la acessado em todas as disciplinas e 29,8\% em nenhuma delas (tabela 3).

A tabela 4 mostra que o hábito de leitura foi considerado crucial para a formação pessoal por 45,6\% dos participantes, para o desempenho acadêmico $(60,5 \%)$, para a elaboração da escrita $(69,3 \%)$ e para o desenvolvimento do raciocínio (47,4\%). Foi observado que, à medida

\begin{tabular}{|c|c|c|c|c|c|c|c|c|c|c|}
\hline \multirow{3}{*}{$\begin{array}{l}\text { Etapa } \\
\text { Total } \\
\text { E-books }\end{array}$} & \multicolumn{2}{|c|}{$2^{a}$} & \multicolumn{2}{|c|}{$4^{a}$} & \multicolumn{2}{|c|}{$6^{a}$} & \multicolumn{2}{|c|}{$8^{a}$} & \multicolumn{2}{|c|}{ Total } \\
\hline & \multicolumn{2}{|c|}{28} & \multicolumn{2}{|c|}{31} & \multicolumn{2}{|c|}{33} & \multicolumn{2}{|c|}{22} & \multicolumn{2}{|c|}{114} \\
\hline & $\mathrm{n}$ & $\%$ & $\mathrm{n}$ & $\%$ & $\mathrm{n}$ & $\%$ & $\mathrm{n}$ & $\%$ & $\mathrm{n}$ & $\%$ \\
\hline Diariamente & 2 & 7,1 & 1 & 3,2 & 3 & 9,0 & 0 & 0 & 6 & 5,26 \\
\hline Algumas vezes por semana & 5 & 17,85 & 3 & 9,7 & 4 & 12,1 & 4 & 18,1 & 16 & 11,1 \\
\hline Somente aos fins de semana & 2 & 7,1 & 1 & 3,2 & 1 & 3,0 & 1 & 4,5 & 5 & 4,4 \\
\hline Raramente & 7 & 25 & 16 & 51,6 & 15 & 45,45 & 11 & 50 & 49 & 42,9 \\
\hline Nunca & 11 & 39,3 & 10 & 32,25 & 10 & 30,3 & 5 & 22,7 & 36 & 31,6 \\
\hline $\begin{array}{l}\text { Artigos científicos pelo } \\
\text { Scielo }\end{array}$ & $\mathrm{n}$ & $\%$ & $\mathrm{n}$ & $\%$ & $\mathrm{n}$ & $\%$ & $\mathrm{n}$ & $\%$ & $\mathrm{n}$ & $\%$ \\
\hline Diariamente & 0 & 0 & 1 & 3,2 & 2 & 6,0 & 4 & 18,1 & 7 & 6,1 \\
\hline Algumas vezes por semana & 1 & 3,6 & 14 & 45,1 & 23 & 69,7 & 18 & 81,8 & 56 & 49,1 \\
\hline Somente aos fins de semana & 2 & 7,1 & 4 & 12,9 & 2 & 6,0 & 0 & 0 & 8 & 7 \\
\hline Raramente & 15 & 53,6 & 12 & 38,7 & 6 & 6 & 0 & 0 & 33 & 28,9 \\
\hline Nunca & 9 & 32,1 & 0 & 0 & 0 & 18,2 & 0 & 0 & 9 & 7,9 \\
\hline $\begin{array}{l}\text { Dissertações/teses pela } \\
\text { biblioteca virtual da USP }\end{array}$ & $\mathrm{n}$ & $\%$ & $\mathrm{n}$ & $\%$ & $\mathrm{n}$ & $\%$ & $\mathrm{n}$ & $\%$ & $\mathrm{n}$ & $\%$ \\
\hline Diariamente & 0 & 0 & 0 & 0 & 0 & 0,0 & 0 & 0 & 0 & 0 \\
\hline Algumas vezes por semana & 2 & 7,1 & 1 & 3,2 & 6 & 18,1 & 9 & 40,9 & 18 & 15,8 \\
\hline Somente aos fins de semana & 1 & 3,6 & 2 & 6,45 & 1 & 3,0 & 0 & 0 & 4 & 3,5 \\
\hline Raramente & 12 & 42,85 & 19 & 61,3 & 19 & 57,6 & 10 & 45,45 & 60 & 52,6 \\
\hline Nunca & 13 & 46,4 & 9 & 29 & 7 & 21,2 & 3 & 13,6 & 32 & 28 \\
\hline
\end{tabular}




\begin{tabular}{|c|c|c|c|c|c|c|c|c|c|c|}
\hline $\begin{array}{l}\text { Revistas eletrônicas pela } \\
\text { biblioteca virtual da USP }\end{array}$ & $\mathrm{n}$ & $\%$ & $\mathrm{n}$ & $\%$ & $\mathrm{n}$ & $\%$ & $\mathrm{n}$ & $\%$ & $\mathrm{n}$ & $\%$ \\
\hline Diariamente & 0 & 0 & 0 & 0 & 0 & 0,0 & 0 & 0 & 0 & 0 \\
\hline Algumas vezes por semana & 4 & 14,3 & 1 & 3,2 & 6 & 18,1 & 7 & 31,8 & 18 & 15,8 \\
\hline Somente aos fins de semana & 0 & 0 & 2 & 6,45 & 0 & 0,0 & 0 & 0 & 2 & 1,75 \\
\hline Raramente & 7 & 25 & 17 & 54,7 & 15 & 11 & 50 & 50 & 50 & 43,85 \\
\hline Nunca & 17 & 60,7 & 11 & 35,5 & 12 & 4 & 18,1 & 18,1 & 44 & 38,6 \\
\hline $\begin{array}{l}\text { Acessa revistas } \\
\text { eletrônicas pelo portal } \\
\text { de periódicos da Capes }\end{array}$ & $\mathrm{n}$ & $\%$ & $\mathrm{n}$ & $\%$ & $\mathrm{n}$ & $\%$ & $\mathrm{n}$ & $\%$ & $\mathrm{n}$ & $\%$ \\
\hline Diariamente & 0 & 0 & 0 & 0 & 0 & 0 & 0 & 0 & 0 & 0 \\
\hline Algumas vezes por semana & 0 & 0 & 0 & 0 & 3 & 9 & 3 & 13,6 & 6 & 5,3 \\
\hline Somente aos fins de semana & 1 & 3,6 & 1 & 3,2 & 2 & 6 & 0 & 0 & 4 & 3,5 \\
\hline Raramente & 5 & 17,85 & 14 & 45,1 & 20 & 60,6 & 13 & 59 & 52 & 45,6 \\
\hline Nunca & 22 & 78,6 & 16 & 51,6 & 8 & 24,3 & 6 & 27 & 52 & 45,6 \\
\hline $\begin{array}{l}\text { Bibliografia } \\
\text { obrigatória das } \\
\text { disciplinas que cursa } \\
\text { através do sistema } \\
\text { Júpiter/USP }\end{array}$ & $\mathrm{n}$ & $\%$ & $\mathrm{n}$ & $\%$ & $\mathrm{n}$ & $\%$ & $\mathrm{n}$ & $\%$ & $\mathrm{n}$ & $\%$ \\
\hline $\begin{array}{l}\text { Em todas as disciplinas do } \\
\text { curso }\end{array}$ & 9 & 32,1 & 6 & 19,38 & 13 & 39,4 & 7 & 31,8 & 35 & 30,7 \\
\hline Somente nas do ciclo básico & 2 & 7,1 & 1 & 3,2 & 1 & 3 & 1 & 4,5 & 5 & 4,4 \\
\hline $\begin{array}{l}\text { Somente nas do ciclo } \\
\text { específico }\end{array}$ & 3 & 10,7 & 5 & 16,1 & 13 & 39,4 & 8 & 36,4 & 29 & 25,4 \\
\hline $\begin{array}{l}\text { Somente nas de prática } \\
\text { profissionalizante }\end{array}$ & 0 & 0 & 3 & 9,7 & 3 & 9 & 5 & 22,7 & 11 & 9,6 \\
\hline $\begin{array}{l}\text { Em nenhuma disciplina do } \\
\text { curso }\end{array}$ & 14 & 50 & 16 & 51,6 & 3 & 9 & 1 & 4,5 & 34 & 29,8 \\
\hline
\end{tabular}

Tabela 3 - Acesso a recursos de leitura.

que os alunos vão progredindo nas etapas do curso, a importância dada à leitura parece progredir também.

A tabela 5 mostra que a maioria dos estudantes respondeu que o hábito de leitura é incentivado pelos docentes em todas as disciplinas do curso $(72,8 \%)$. No entanto, quando se perguntou se é possível manter o hábito de leitura cursando a universidade em tempo integral, 51,7\% responderam que raramente.

Quando questionada(s) qual(is) estratégia(s) para melhorar o hábito de leitura, a maioria dos participantes destacou a disponibilidade de tempo $(47,4 \%)$. 


\begin{tabular}{|c|c|c|c|c|c|c|c|c|c|c|}
\hline Etapa & \multicolumn{2}{|c|}{$2^{a}$} & \multicolumn{2}{|c|}{$4^{a}$} & \multicolumn{2}{|c|}{$6^{a}$} & \multicolumn{2}{|c|}{$8^{a}$} & \multicolumn{2}{|c|}{ Total } \\
\hline Total & \multicolumn{2}{|c|}{28} & \multicolumn{2}{|c|}{31} & \multicolumn{2}{|c|}{33} & \multicolumn{2}{|c|}{22} & \multicolumn{2}{|c|}{114} \\
\hline Para a formação pessoal & $\mathrm{n}$ & $\%$ & $\mathrm{n}$ & $\%$ & $\mathrm{n}$ & $\%$ & $\mathrm{n}$ & $\%$ & $\mathrm{n}$ & $\%$ \\
\hline Crucial & 8 & 28,6 & 16 & 51,6 & 15 & 45,45 & 13 & 59 & 52 & 45,6 \\
\hline Muito importante & 10 & 35,7 & 10 & 32,25 & 14 & 42,4 & 8 & 36,4 & 42 & 36,8 \\
\hline Importante & 10 & 35,7 & 5 & 16,1 & 3 & 9 & 1 & 4,5 & 19 & 16,7 \\
\hline Pouco importante & 0 & 0 & 0 & 0 & 0 & 0 & 0 & 0 & 0 & 0 \\
\hline Insignificante & 0 & 0 & 0 & 0 & 0 & 0 & 0 & 0 & 0 & 0 \\
\hline $\begin{array}{l}\text { Para o desempenho } \\
\text { acadêmico }\end{array}$ & $\mathrm{n}$ & $\%$ & $\mathrm{n}$ & $\%$ & $\mathrm{n}$ & $\%$ & $\mathrm{n}$ & $\%$ & $\mathrm{n}$ & $\%$ \\
\hline Crucial & 13 & 46,4 & 21 & 67,7 & 21 & 63,6 & $14^{*}$ & 63,6 & 69 & 60,5 \\
\hline Muito importante & 10 & 35,7 & 7 & 22,6 & 10 & 30,3 & 6 & 27,3 & 33 & 28,9 \\
\hline Importante & 5 & 17,85 & 3 & 9,7 & 2 & 6 & 0 & 0 & 10 & 8,8 \\
\hline Pouco importante & 0 & 0 & 0 & 0 & 0 & 0 & 0 & 0 & 0 & 0 \\
\hline Insignificante & 0 & 0 & 0 & 0 & 0 & 0 & 0 & 0 & 0 & 0 \\
\hline $\begin{array}{l}\text { Para a elaboração da } \\
\text { escrita }\end{array}$ & $\mathrm{n}$ & $\%$ & $\mathrm{n}$ & $\%$ & $\mathrm{n}$ & $\%$ & $\mathrm{n}$ & $\%$ & $\mathrm{n}$ & $\%$ \\
\hline Crucial & 19 & 67,85 & 23 & 74,2 & 20 & 60,6 & 17 & 77,3 & 79 & 69,3 \\
\hline Muito importante & 4 & 14,3 & 8 & 25,8 & 12 & 36,4 & 5 & 22,7 & 29 & 25,4 \\
\hline Importante & 5 & 17,85 & 0 & 0 & 1 & 3 & 0 & 0 & 6 & 5,3 \\
\hline Pouco importante & 0 & 0 & 0 & 0 & 0 & 0 & 0 & 0 & 0 & 0 \\
\hline Insignificante & 0 & 0 & 0 & 0 & 0 & 0 & 0 & 0 & 0 & 0 \\
\hline $\begin{array}{l}\text { Internet (Site de agência de } \\
\text { notícias) }\end{array}$ & 17 & 60,7 & 23 & 74,1 & 21 & 63,6 & 13 & 59 & 74 & 65,6 \\
\hline $\begin{array}{l}\text { Para o desenvolvimento } \\
\text { do raciocínio }\end{array}$ & $\mathrm{n}$ & $\%$ & $\mathrm{n}$ & $\%$ & $\mathrm{n}$ & $\%$ & $\mathrm{n}$ & $\%$ & $\mathrm{n}$ & $\%$ \\
\hline Crucial & 12 & 42,85 & 16 & 50 & 16 & 48,5 & 10 & 45,45 & 54 & 47,4 \\
\hline Muito importante & 9 & 32,1 & 11 & 35,5 & 11 & 33,3 & 9 & 40,9 & 40 & 35 \\
\hline Importante & 7 & 25 & 4 & 12,9 & 6 & 18,1 & 3 & 13,6 & 20 & 17,5 \\
\hline Pouco importante & 0 & 0 & 0 & 0 & 0 & 0 & 0 & 0 & 0 & 0 \\
\hline Insignificante & 0 & 0 & 0 & 0 & 0 & 0 & 0 & 0 & 0 & 0 \\
\hline
\end{tabular}

Tabela 4 - Opinião sobre hábito de leitura. *Dois participantes não responderam. 


\begin{tabular}{|c|c|c|c|c|c|c|c|c|c|c|}
\hline \multirow{3}{*}{$\begin{array}{l}\text { Etapa } \\
\text { Total } \\
\text { O hábito de leitura } \\
\text { é incentivado pelos } \\
\text { docentes do curso? }\end{array}$} & \multicolumn{2}{|c|}{$2^{a}$} & \multicolumn{2}{|c|}{$4^{a}$} & \multicolumn{2}{|c|}{$6^{a}$} & \multicolumn{2}{|c|}{$8^{a}$} & \multicolumn{2}{|c|}{ Total } \\
\hline & \multicolumn{2}{|c|}{28} & \multicolumn{2}{|c|}{31} & \multicolumn{2}{|c|}{33} & \multicolumn{2}{|c|}{22} & \multicolumn{2}{|c|}{114} \\
\hline & $\mathrm{n}$ & $\%$ & $\mathrm{n}$ & $\%$ & $\mathrm{n}$ & $\%$ & $\mathrm{n}$ & $\%$ & $\mathrm{n}$ & $\%$ \\
\hline $\begin{array}{l}\text { Em todas as disciplinas do } \\
\text { curso }\end{array}$ & 21 & 75 & 23 & 74,2 & 22 & 66,7 & 17 & 77,3 & 83 & 72,8 \\
\hline Somente nas do ciclo básico & 4 & 14,3 & 0 & 0 & 1 & 3 & 1 & 4,5 & 6 & 5,3 \\
\hline $\begin{array}{l}\text { Somente nas do ciclo } \\
\text { específico (fonoaudiologia) }\end{array}$ & 3 & 10,7 & 8 & 25,8 & 9 & 27,3 & 4 & 18,1 & 24 & 21 \\
\hline $\begin{array}{l}\text { Somente nas de prática } \\
\text { profissionalizante (estágio) }\end{array}$ & 0 & 0 & 0 & 0 & 1 & 3 & 0 & 0 & 1 & 0,9 \\
\hline $\begin{array}{l}\text { É possível manter o } \\
\text { hábito de leitura cursando } \\
\text { a Universidade em tempo } \\
\text { integral? }\end{array}$ & $\mathrm{n}$ & $\%$ & $\mathrm{n}$ & $\%$ & $\mathrm{n}$ & $\%$ & $\mathrm{n}$ & $\%$ & $\mathrm{n}$ & $\%$ \\
\hline Sim & 11 & 39,3 & 10 & 32,25 & $8^{*}$ & 24,3 & 5 & 22,7 & 34 & 29,8 \\
\hline Raramente & 15 & 53,6 & 17 & 54,8 & 12 & 36,4 & 15 & 68,1 & 59 & 51,7 \\
\hline Não & 2 & 7,1 & 4 & 12,9 & 12 & 36,4 & 2 & 9 & 20 & 17,5 \\
\hline
\end{tabular}

Tabela 5 - Opinião sobre a relação entre hábito de leitura e formação profissional

\section{Conclusão}

Embora o hábito de leitura tenha sido considerado crucial pelos estudantes, grande parte deles encara manter tal hábito durante a graduação como um desafio, em consequência da dificuldade em organizar o tempo para as atividades acadêmicas.

\section{Referências Bibliográficas}

BERTICELLI, I. A. \& SCHIAVINI, D. P. "Significados da Pragmática Linguística na Formação de Leitores". Educasãa \& Realidade, Porto Alegre, vol. 38, n. 2, pp. 571-586, abr.jun. 2013.

BRIDON, J. \& NEITZEL, A. A. "Competências Leitoras no Saeb: Qualidade da Leitura na Educação Básica". Educação \& Realidade, Porto Alegre, vol. 39, n. 2, pp. 437-462, 2014.

CARAVANTES, G. R. Leitura Dinâmica e Aprendizagem. 2 ed. Porto Alegre: Ed. Age, 2006.

CARDOSO, A. L. M. S.; FRANÇA, C. M. \& MARIANI, A. M. M. "Formação de Usuários para
Competência Informacional em Ambientes Virtuais". XVIII Seminário Nacional de Bibliotecas Universitárias (SNBU), 2014.

FERREIRA, A. G. G. \& CAREGNATO, S. E. "Visibilidade de Revistas Científicas: um Estudo no Portal de Periódicos Científicos da Universidade Federal do Rio Grande do Sul". TransInformação, Campinas, vol. 26, n. 2, pp. 177-190, 2014.

FRANCO, S. A. P \& MOLINARI, A. C. "A Importância da Leitura e as Concepções dos Graduandos do Curso de Extensão Leitura e Escrita na Universidade: uma Prática Transformadora". Inter-Ação, Goiânia, vol. 38, n. 3, pp. 663-675, 2013.

GONZÁLEZ, E. A.; GOMES, I. M. A. M. \& ANDRADE, V. T. A. "Discursos Estratégicos e Ações para a Comunicação Científica de Acesso Livre: Estudo Comparado entre Brasil e Espanha". Comunicação \& Inovação, São Caetano do Sul, vol. 15, n. 28, pp. 20-31, 2014.

HUSSEIN, C. L. "Avaliação de Treino de Leitura Compreensiva e Crítica: Estudo com Universitários". Revista Semestral da Associação Brasileira de Psicologia Escolar e 
Educacional (ABRAPEE), vol. 12, n. 2, p. 401-411, 2008.

LAKATOS, E. M. \& MARCONI, M. A. Metodologia do Trabalho Científico: Procedimentos Básicos, Pesquisa Bibliográfica, Projeto e Relatório, Publicações e Trabalhos Científicos. São Paulo: Atlas, 2007.

LARA CAMPOS, L. F.; SILVA FILHO, N.; CAMPOS, P. R. \& ROCHA, R. L. "Caracterização dos Alunos de Psicologia da USF/Itatiba: Características, Opiniões e Expectativas". Psico-USF, vol. 1, n. 2, pp. 57-82, 1996.

MARTINS, M. E. O. \& SÁ, C. M. "Ser Leitor no Século XXI - Importância da Compreensão na Leitura para o Exercício Pleno de uma Cidadania Responsável e Activa". Saber (e) Educar, vol. 13, pp. 235-245, 2008.

MAZZA, D. "Por uma Sociologia da Leitura". Educ. Soc., Campinas:, vol. 34, n. 123, pp. 621-624, 2013.

OLIVEIRA, K. L. \& SANTOS, A. A. A. \& PRIMI, R. "Estudo das Relações entre Compreensão em Leitura e Desempenho em Disciplinas na Universidade". Revista Interaşão em Psicologia, vol. 7, n. 1, pp. 19-25, 2003.

OLIVEIRA, R. Á. M. \& OLIVEIRA, K. L. "Leitura e Condições de Estudo em Universitários Ingressantes". Psic: Revista de Psicologia da Vetor Editora, vol. 8, n. 1, pp. 51-59, 2007.

ORTELLADO, P. "As Políticas Nacionais de Acesso à Informação Científica". Lünc em Revista, Rio de Janeiro, vol. 4, n. 2, pp. 186-195, 2008.

OSWALD, M. L. \& ROCHA, S. L. A. da. "Sobre Juventude e Leitura na "Idade Mídia": Implicações para Políticas e Práticas Curriculares". Educar em Revista, Curitiba, vol. 47, pp. 267-283, 2013.

PIRES, E. A. N. "A Importância do Hábito da Leitura na Universidade". Revista ACB: Biblioteconomia em Santa Catarina, vol. 2, n. 17, pp. 365-381, 2012.

ROHENKOHL, G. "Pelo Livre Acesso ao Conhecimento". Psico, vol. 45, n. 1, p. 6, 2014.

SILVEIRA, R. M. H. "A Leitura e Seus Poderes: um Olhar sobre Dois Programas Nacionais de Incentivo à Leitura". Educar em Revista, Curitiba, pp. 103-120, 2010.

WATKINS, M. W. \& COFFEY, D. Y. "Reading Motivation: Multidimensional and Indeterminate'. Foumal of Education Psychology, vol. 96, n. 1, pp. 110-118, 2004. 